\title{
PREVALÊNCIA DE FATORES DE RISCO DE NATUREZA MODIFICÁVEL PARA A OCORRÊNCIA DE DIABETES MELLIT US TIPO 2
}

\author{
Prevalence of risk factors that can be modified into the occurrence \\ of Type 2 Diabetes \\ Predominio de factores de riesgo posibles de modificar en el \\ acontecimiento de Diabetes tipo 2
}

Rosa Maria Fernandes Vilarinho ${ }^{1}$
Priscila Katzer Thiré

\author{
Márcia Tereza Luz Lisboa² \\ Priscila Vieira França ${ }^{4}$
}

\section{RESUMO}

Estudo transversal realizado com estudantes universitários do curso de Enfermagem para identificação de fatores de risco para 0 diabetes tipo 2. Em relação ao nível de risco: $65 \%$ apresentavam risco baixo, $27 \%$, risco zero, enquanto $7,5 \%$ exibiam risco alto. A história familiar de diabetes e hipertensão foi identificada em $45 \%$ da amostra. A prevalência de sedentarismo foi igual a $75 \%$; de sobrepeso, igual a $15 \%$, e de obesidade (IMC e $\geq 25 \mathrm{~kg} / \mathrm{m}^{2}$ ), igual a $5 \%$. Verificaram-se ainda um menor consumo de alimentos ricos em fibras e 0 aumento do consumo de alimentos ricos em gorduras. Os dados levantados apontaram para a necessidade de investimentos em ações de promoção à saúde dentro da própria unidade de formação, como a criação de espaços próprios destinados à valorização da aquisição de novas práticas de saúde, através da ênfase à prática da atividade física, e incentivo à aquisição de hábitos alimentares saudáveis

Palavras-chave: Diabetes Mellitus. Enfermagem. Saúde do Trabalhador. Fatores de risco.

\begin{abstract}
Cross study carried out near nursing students willing to identify the most prevalent risk factors for type 2 diabetes. Regarding the level of risk: $65 \%$ presented low risk, $27 \%$ none, while 7,5 $\%$ was showing high risk. The history close to diabetes and high blood pressure was identified in $45 \%$ of the sample. The predominance of: sedentary lifestyle was $75 \%$; of equal surcharge to $15 \%$ and obesity (IMC e" $25 \mathrm{~kg} / \mathrm{m2}$ ) equal to 5 $\%$. A low consumption of food rich in fibers happened and the increase of the consumption of food rich in fat. The gathered data pointed to the need of investments in actions in health promotion inside the formation unity itself, like the creation of own spaces to increase the value of that, as acquisition of new practices of health, through the emphasis of the physical activity and incentive to the acquisition of food healthy habits.
\end{abstract}

Keywords: Diabetes mellitus. Nursing. Worker's health. Risk factors.

\section{Resumen}

El estudio enfadado realizado cerca de estudiantes de universidad del curso de enfermería, para la identificación de factores del riesgo para la diabetes tipo 2. En cuanto al nivel de riesgo: el $65 \%$ presentaba bajo el riesgo, el $27 \%$ marco el cero, mientras el 7,5\% mostraba el riesgo alto. La historia cerca de diabetes y hipertensión fue identificada en el $45 \%$ de la muestra. El predominio de: el sedentarismo era el mismo al $75 \%$; de la sobrecarga igual al $15 \%$, y de la obesidad (CMI $e^{\prime \prime} 25 \mathrm{~kg} / \mathrm{m} 2$ ) igualan al $5 \%$. Menos consumo de la comida rica pasó todavía en fibras y el aumento del consumo de la comida rica en grasa. Los datos levantados señalaran la necesidad de inversiones en acciones de promoción a la salud dentro de la misma unidad de formación, como la creación de propios espacios destinados, el aumento del valor de la adquisición de nuevas prácticas de la salud, por el énfasis la práctica de la actividad física y incentivo a la adquisición de alimenticios saludables.
Palabras clave: La diabetes mellitas. Enfermería. Salud del trabajador. Factores de riesgo. 


\section{INTRODUÇÃO}

Trata-se de uma pesquisa cadastrada na linha de pesquisa " 0 aluno de enfermagem frente às questões de trabalho" do Núcleo de Pesquisa Enfermagem e Saúde do Trabalhador (NUPENST) do Departamento de Enfermagem de Saúde Pública (DESP) da Escola de Enfermagem Anna Nery (EEAN) da Universidade Federal do Rio de Janeiro (UFRJ).

As doenças crônicas não transmissiviveis (DCNT) têm-se constituído como um importante problema de saúde pública ${ }^{1}$. Já de longa data a literatura aponta 0 importante papel exercido por fatores de risco de natureza reversível, tais como 0 sedentarismo, a obesidade, 0 tabagismo, a hipertensão - e seu manejo inadequado e a hipercolesterolemia².

A prevenção primária destas ocorrências deve privilegiar esforços para a correção dos fatores de risco de natureza reversível ou modificável, nas diversas faixas etárias ${ }^{3}$, inclusive com grupos etários mais jovens. Essa necessidade se dá primeiramente porque grande parcela dos hábitos de vida do indivíduo adulto é assimilada ainda na infância e adolescência, e segundo porque a ocorrência de DCNT, como, por exemplo, 0 diabetes tipo 2, tem aumentado em faixas etárias cada vez mais precoces ${ }^{1,4,5}$.

Um exemplo de situação que ilustra a relevância de ações neste sentido é a constatação da importante ascensão nas taxas de sobrepeso e obesidade entre crianças e adolescentes, e dos achados que relacionam as ocorrências nesta fase com o desenvolvimento da síndrome metabólica na idade adulta. Esse dado é potencialmente relevante quando se considera que $40 \%$ das crianças obesas tendem a ser tornar adolescentes obesos, e que $75 \%$ destes adolescentes tendem a se tornar adultos obesos. Isto ainda, sem levar em conta, a tendência natural para 0 ganho de peso durante a fase da vida adulta, 0 que é estimado em 3 a $5 \mathrm{~kg}$ por década até a sexta década de vida, quando o peso então se estabiliza e tende a diminuir ${ }^{4,5,6}$.

No desenvolvimento do diabetes mellitus tipo 2, podemos, a partir do Consenso Brasileiro de Diabetes ${ }^{7,8}$, relacionar cinco fatores de risco não modificáveis: a idade, a história familiar de diabetes mellitus, a ocorrência do diabetes gestacional prévio, a história de macrossomia fetal e a ocorrência de abortos de repetição ou mortalidade peri-natal.

Entre os fatores de risco de natureza modificável se encontram aqueles passíveis de intervenções, ou controle, como: 0 excesso de peso pelo Índice de Massa Corporal (IMC) - IMC $\geq 25 \mathrm{Kg} / \mathrm{m}^{2}$, o sedentarismo, o nível de hdl-c baixo e/ou de triglicérides elevados, a hipertensão arterial e uso de medicação hiperglicemiante (por exemplo, corticosteróides, tiazídicos, betabloqueadores $)^{8}$. Esses fatores devem, portanto, ser motivo de atenção especial por parte dos indivíduos empenhados em seu autocuidado.

Uma das causas mais freqüentes associadas à ocorrência de fatores de risco de natureza reversível é o erro alimentar. Um padrão alimentar inadequado é um elemento-chave para uma série de condiç̧ões de risco. Em princípio, a adoção de uma dieta balanceada é uma forma de precaução bastante importante. Mas, além deste cuidado, se fazem necessários a adesão à prática de atividade física e o esforço para o controle do peso $0^{1,6,8,9}$.

Em 2003, o Ministério da Saúde concebeu um material educativo bastante interessante, além de simples e objetivo. A cartilha intitulada "Dez passos para uma alimentação saudável" 10 enumera dez cuidados considerados importantes para 0 alcance de um padrão de vida considerado saudável. Apesar de o título direcionar a questão da alimentação saudável, o texto também traz recomendações acerca da importância da prática de exercícios e do controle do peso.

Partindo do princípio que importantes fatores de risco para ocorrências de DCNT podem ser identificados mesmo em adolescentes e adultos jovens, 0 estudo teve como objetivo identificar a prevalência de fatores de risco de natureza modificável para a ocorrência de diabetes mellitus tipo 2 entre estudantes de Enfermagem.

Cabe ressaltar que os sujeitos desta pesquisa são estudantes do curso de graduação em Enfermagem, ou seja, futuros enfermeiros que em breve estarão no mercado de trabalho. Como pesquisadoras da área da saúde do trabalhador, muito nos interessam os compor tamentos de risco desse grupo de trabalhadores. Identificando problemas advindos de riscos encontrados, muitas vezes no próprio trabalho, especificamente na organização do trabalho ou nas condições do trabalho de alunos e profissionais, poderemos, a partir do diagnóstico feito, investir em área de promoção da saúde contribuindo para 0 decréscimo no adoecimento dos trabalhadores de enfermagem.

\section{MÉTODOS}

Trata-se de um estudo transversal, realizado com 40 estudantes universitários do 40 período do curso de enfermagem de uma instituição pública localizada na cidade do Rio de Janeiro. A construção da amostra foi intencional, sendo a mesma constituída por estudantes que estavam presentes nas aulas durante o período de coleta de dados, compreendido entre os meses de setembro e outubro de 2006. 0 projeto foi aprovado pelo Comitê de Ética em Pesquisa da Escola de Enfermagem Anna Nery (EEAN)/Hospital Escola São Francisco de Assis (HESFA) em maio de 2006. Atendendo a resolução 196/96, após a aceitação de inclusão na pesquisa, os participantes assinaram o termo de consentimento livre e esclarecido. A coleta de dados foi realizada exclusivamente pelas autoras Priscila Katzer e Priscila Vieira, após treinamento, e aconteceu em espaço próprio e reservado, localizado no HESFA, aproveitando-se o intervalo de aulas ministradas durante 0 turno da tarde, no período citado anteriormente.

Foram variáveis do Estudo: 1) dados biológicoconstitucionais: sexo, idade; 2) dados de natureza antropométrica: peso, altura, IMC, circunferência abdominal; 3) dados da história de saúde: história prévia de ocorrências de sobrepeso e obesidade, alterações do perfil lipídico e níveis elevados de pressão arterial; 4) dados da história familiar: freqüência de casos de diabetes e hiper tensão entre parentes de primeiro e segundo graus, e referência de prática de atividade física entre familiares; 5) hábitos de vida relacionados ao estilo 
de vida individual: padrão de atividade física, prática do tabagismo e padrão alimentar.

Para a coleta de dados foram utilizados instrumentos, distribuídos da seguinte forma: a) do is instrumentos de coleta de dados para a identificação das variáveis sócio-demográficas e de estilo de vida e b) um instrumento para coleta de dados antropométricos.

Os instrumentos de coleta de dados para a identificação das variáveis sócio-demográficas e de estilo de vida constam de duas partes, a saber: Instrumento no 1 - formulário desenvolvido para o estudo, dividido em quatro itens: identificação, medidas antropométricas, estado de saúde e identificação de hábitos de vida relacionados ao estilo de vida (prática do autocuidado); Instrumento no 2 - questionário desenvolvido pela Harvard-Joslin-SBD, intitulado "É possível você ter diabetes e não saber?".

Para coleta dos dados antropométricos, utilizamos a balança antropométrica, estadiômetro e fita métrica inflexível.

Para a organização dos dados colhidos, foram adotados os seguintes procedimentos:

- Procedimentos para a identificação dos dados antropométricos.

Procedimentos adotados para a verificação das medidas antropométricas, circunferência abdominal e nível de adequação do peso:

\section{a)Peso corporal (Kg)}

0 peso corporal foi determinado através de uma mesma balança antropométrica, com precisão de $0,05 \mathrm{Kg}$, escala de 0 a $150 \mathrm{Kg}$ e da marca FILIZOLA. As recomendações que se fizeram para medir o peso corporal se restringiram à solicitação de esvaziamento de bolsos, retirada de sapatos, jalecos e agasalhos, buscando-se garantir o uso do menor número possível de roupas.

\section{b) Estatura corporal (cm)}

A estatura $(\mathrm{cm})$ foi obtida utilizando-se o estadiômetro correspondente à balança antropométrica, com precisão de $0,1 \mathrm{~cm}$. A estatura foi determinada colocando-se 0 indivíduo na posição or tostática (em pé), completamente reto e com os pés juntos.

\section{c) Circunferência abdominal (cm)}

Para a verificação da circunferência abdominal, foi utilizada fita métrica com precisão de $0,1 \mathrm{~cm}$, com escala de 0 a 150 $\mathrm{cm}$. Para a efetuação da medida, foi solicitado ao indivíduo que se posicionasse de pé. Para identificação do valor, foi considerada a menor medida da circunferência no nível da cicatriz umbilical, no final do movimento expiratório ${ }^{11}$.

A identificação do nível de risco, associado ao valor da circunferência abdominal, foi obtida com base nos valores sugeridos pelo Consenso Latino-Americano de Obesidade ${ }^{12,13}$, que assinala uma indicação de risco aumentado para patologias como o diabetes mellitus tipo 2 e doenças cardiovasculares valores a partir de $102 \mathrm{~cm}$ para homens e $88 \mathrm{~cm}$ para mulheres.

d) Niveis de adequação do peso

A adequação do peso à estatura foi identificada através da utilização do cálculo do índice de Quetelet, que se obtém da divisão do peso em quilos (P) pela altura em metros (A) elevada ao quadrado. $I M C=P / A^{2} 4,6$.

A identificação das categorias de classificação de adequação do peso à estatura foi feita com base nos critérios estabelecidos pela Organização Mundial de Saúde para adultos de 20 anos ou mais, independente do sexo e idade (Quadro I) 4,6,13. Segundo esta classificação considera-se: desnutrição grau 1 valores de IMC de 17 a 18,4; peso saudável valores de IMC de 18,5 a 24,9; sobrepeso valores de IMC de 25 a 29,9; obesidade grau 1 valores de IMC de 30 a 34,9; obesidade grau 2 valores de IMC de 35 a 39,9; e obesidade grau 3 valores de IMC de 40 ou superior.

- Procedimentos adotados para verificação dos níveis de risco para 0 desenvolvimento do diabetes mellitus tipo 2

Para a estratificação da amostra, em níveis de risco, foi utilizado um instrumento desenvolvido pela Harvard-JoslinSBD, intitulado "É possível você ter diabetes e não saber?"14, que, neste estudo, é denominado "Instrumento n-2". Avaliação de 0 a 2 pontos indica risco igual a zero; a avaliação de 3 a 9 pontos indica baixo risco; e, finalmente, a avaliação de 10 ou mais pontos indica alto risco ${ }^{14}$.

- Procedimentos adotados para identificação de padrão alimentar considerado adequado.

Para efeito de identificação de padrão alimentar adequado foram consideradas as indicações da Cartilha intitulada "Dez passos para uma alimentação saudável" ${ }^{10}$, que apresenta as seguintes recomendações: PASSO № 1 Aumente e varie 0 consumo de frutas, legumes e verduras; coma-os 5 vezes por dia; PASSO № 2 Coma feijão pelo menos 1 vez por dia, no mínimo 4 vezes por semana; PASSO № 3 Reduza o consumo de alimentos gordurosos, como carnes com gordura aparente, salsicha, mortadela, frituras e salgadinhos, para no máximo 1 vez por semana; PASSO № 4 Reduza o consumo de sal; PASSO № 5 Faça pelo menos 3 refeições e 1 lanche por dia. Não pule as refeições; PASSO № 6 Reduza o consumo de doces, bolos, biscoitos e outros. PASSO No 7 Reduza o consumo de álcool e refrigerantes. Evite o consumo diário; PASSO № 8 Aprecie a sua refeição. Coma devagar; PASSO № 9 Mantenha o seu peso dentro de limites saudáveis; PASSO № 10 Seja ativo. "Acumule 30 minutos de atividades físicas todos os dias."

- Procedimentos adotados para identificação de padrão de atividade física considerado adequado.

Para efeito de identificação do padrão adequado de realização de atividades físicas, foi utilizada a recomendação indicada no Plano de Reorganização da Atenção à Hipertensão e ao Diabetes Mellitus ${ }^{13}$, que sugere como prática adequada a ser empregada nas ações de prevenção ao diabetes a realização de 30 minutos de atividade física diária (ou pelo menos três vezes na semana).

Na tabulação dos dados, foi utilizado o programa EPI-INFOversão 6.04. Para o tratamento de dados, utilizou-se de técnica estatística descritiva simples, compreendida pela distribuição de freqüências absolutas e relativas. 


\section{RESULTADOS}

\section{Caracterização da a mostra}

A amostra foi constituída de 40 acadêmicos de enfermagem, com idades entre 18 e 28 anos, sendo $35(87,5 \%)$ do sexo feminino e $5(12,5 \%)$ do sexo masculino. Destes, 21 (52,5\%) participantes se encontravam na faixa etária entre 20 e 21 anos, $9(22,5 \%)$ na faixa de 22 a 23 anos, $6(15 \%)$ na faixa de 18 a 19 anos, 2 (5\%) na faixa de 24 a 25 anos, e 2 (5\%) na faixa de 26 a 28 anos.

\section{Padrão de risco para desenvolvimento do diabetes mellitus tipo 2}

Quando submetidos a instrumento para a avaliação de risco de desenvolvimento de diabetes tipo 2, 3(7,5\%) estudantes foram identificados como exibindo risco alto, $26(65 \%)$ estudantes exibindo risco baixo e 11 (27,5\%) estudantes com risco zero para o momento.

\section{Antecedentes familiares para diabetes e hipertensão}

Em relação aos antecedentes familiares, 23 (57,5\%) estudantes referiram casos de diabetes mellitus na família, 30 (75\%) estudantes referiram casos de hiper tensão e 18 (45\%) estudantes, casos diabetes e hiper tensão simultaneamente, 0 que já revela um importante fator de risco para a doença, justificando ações de educação para o autocuidado.

\section{Ocorrência de fatores de risco de natureza modificável}

No levantamento dos fatores de risco modificáveis, identificou-se que $30(75 \%)$ estudantes eram sedentários, 6 $(15 \%)$ estudantes exibiam circunferência abdominal aumentada, $6(15 \%)$ estudantes apresentavam sobrepeso e 2 $(5 \%)$ estudantes eram obesos. Exibiam peso compatível com a altura $28(70 \%)$ estudantes e peso inferior ao adequado 4 $(10 \%)$ estudantes.

Ao serem interrogados sobre hábitos alimentares, 21 (52,5 $\%$ ) estudantes referiram o consumo diário de frutas frescas, 17 (42,5\%), o consumo diário, ou aumentado, de alimentos gordurosos, $15(37,5 \%)$, de verduras, e apenas $6(15 \%)$ estudantes informaram o consumo diário de feilão. 0 menor consumo de alimentos ricos em fibras e 0 aumento do consumo de alimentos ricos em gorduras acompanham a nova realidade no perfil de consumo da sociedade brasileira, que exibe desde a década de 1970, entre outras características, o "abandono do consumo de uma das preparações mais típicas e comuns a todas às regiões brasileiras - 0 arroz com feilão, combinação nutricionalmente rica e adequada" 6 .

Ao serem analisados os dados referentes à parcela da amostra composta exclusivamente por estudantes com antecedentes familiares para o diabetes mellitus, identificouse que o nível de sedentarismo manteve-se em valor aproximado ao da amostra original, sendo identificado em $69,5 \%$ dos participantes. Já o valor de IMCe $\geq 25 \mathrm{~kg} / \mathrm{m}^{2}$ exibiu prevalência aumentada, sendo registrado em $26 \%$ dos integrantes deste grupo. Em relação aos hábitos alimentares, os niveis de consumo de alimentos gordurosos e de vegetais se mantiveram em valores aproximados ao grupo maior, tendo a ingestão diária de alimentos gordurosos sido identificada em $43 \%$ do grupo, e o consumo de verduras em $39 \%$. Já o consumo diário de frutas decaiu para $17 \%$, enquanto o consumo diário de feijão aumentou, sendo identificado em $39 \%$ destes participantes.

Quando interrogados acerca da história pregressa de sobrepeso ou obesidade, hipercolesterolemia e hipertensão, 6 $(15 \%)$ estudantes confirmaram antecedentes de valores alterados para dosagem do colesterol, $24(60 \%)$ negaram antecedentes desta condição, enquanto $10(25 \%)$ estudantes não souberam responder. Em relação à ocorrência de sobrepeso e/ou obesidade em momentos anteriores, 12 (30\%) estudantes confirmaram esta condição, 27 (67,5\%) estudantes negaram e $1(2,5 \%)$ estudante não soube responder. Interrogados quanto à história pregressa de hiper tensão, $1(2,5 \%)$ estudante referiu história de hiper tensão, $5(37 \%)$ estudantes negaram tal ocorrência, enquanto 5 (37\%) estudantes desconheciam esta informação.

Merece destaque o fato de que, embora o grupo seja formado por adolescentes e adultos jovens, já foi possível observar relatos de alteração do perfil lipídico e dos níveis pressóricos, ocorrências que vem sendo registradas por diversos autores, tanto em países considerados desenvolvidos como aqueles em desenvolvimento $5,6,9,11$

\section{CONSIDERAÇÕES FINAIS}

Diversos estudos relacionam as modificações ocorridas no perfil a limentar e na prática de atividade física das populações no favorecimento de doenças crônicas degenerativas ${ }^{1,6,9,13}$. Estas, por sua vez, tendem a incidir em faixas etárias cada vez mais jovens, inclusive já na fase da infância e adolescênciaja,6,9,11. Se não corrigidos precocemente, a manutenção destes fatores de risco tende a se perpetuar durante a idade adulta, expondo estes indivíduos a um risco aumentado da ocorrência de diabetes e outras doenças metabólicas ${ }^{3,6}$.

No grupo investigado, apesar de a amostra ser caracterizada por indivíduos jovens, com nível de escolaridade elevada, é possível identificar a presença de fatores de risco comportamentais potencialmente modificáveis. Entre estes exibiram maior destaque 0 sedentarismo, 0 alto consumo de alimentos gordurosos e o baixo consumo diário de alimentos ricos em fibras. Índices de sobrepeso e obesidade, apesar de em menor magnitude, também puderam ser evidenciados.

A maior parte dos integrantes desse grupo se caracterizou por exibir um IMC dentro dos valores de normalidade; todavia, a manutenção dos comportamentos citados anteriormente fatalmente os exporá a um aumento destes índices, a natural expectativa de ganho de peso ao longo da vida adulta ${ }^{4,6}$. Não devem ainda ser desprezados os relatos das ocorrências autoreferidas de hipercolesterolemia e hipertensão, elementos indicativos de alterações metabólicas, além da presença da história familiar para diabetes e hipertensão, considerados fatores de risco não modificáveis ${ }^{5}$. 
A manutenção dos fatores de risco identificados entre estes estudantes pode, se não corrigida, contribuir para a ocorrência de uma série de doenças crônicas no futuro. Os dados levantados apontam para a necessidade de investimentos em ações de promoção à saúde não somente voltadas à detecção precoce ${ }^{7}$, mas também ao desenvolvimento de medidas preventivas dentro da própria unidade de formação, como a criação de espaços próprios destinados à valorização da aquisição de novas práticas de saúde, através da ênfase à prática da atividade física, e incentivo à aquisiçãa de hábitos alimentares saudáveis.

Embora não tenha sido objeto desta pesquisa, cabe sugerir para futuros estudos avaliar as condições nas quais os alunos de Enfermagem estão inseridos e de que maneiras as mesmas contribuem para uma vida saudável ou um adoecimento precoce ou previsto.

\section{Referências}

1. Medina MC. Proposta de estratégias de prevenção de doenças crônicas. São Paulo (SP): Centro de Vigilância Epidemiológica Professor Alexandre Vranjac; 2000.33 p.

2. Duncan BB, Schmidt MI, Giugliani ERJ. Medicina ambulatorial: condutas clínicas em atenção primária. Porto Alegre (RS): Artes Médicas; 1990. 495 p.

3. Gabbay M, Cesarini PR, Dib SA. Diabetes melito do tipo 2 na infância e adolescência: revisão da literatura. J Pediatr 2003; 79(3): 201-08.

4. Jorge Filho J. Em busca da saúde ideal. Belo Horizonte (MG): Leitura Saúde; 2001.239p.

5. Farias JM. Orientação para prevenção e controle a da obesidade juvenil: um estudo de caso. [dissertação de mestrado] Florianópolis (SC): Coordenadoria de Pós-graduação em Educação Física/ UFSC; 2005. Disponível em: <http://150.162.90.250/teses/ PGEF0109.pdf.>

6. Ministério da Saúde (BR). Guia alimentar para a população brasileira. Brasilila (DF); 2005.

7. Vilarinho RMF, Lisboa MTL. Assistência de enfermagem na prevenção do diabetes mellitus tipo 2: uma questão da atualidade. Esc Anna Nery Rev Enferm 2005 abr; 9(1): 103 -07.
8. Sociedade Brasileira de Diabetes. Consenso Brasileiro sobre Diabetes. São Paulo (SP); 2001.50 p.

9. Organização Pan-Americana de Saúde-OPAS. Doenças crônicodegenerativas e obesidade: estratégia mundial sobre alimentação saudável, atividade física e saúde. Brasília (DF); 2003. 6 p.

10. Ministério da Saúde (BR). Dez passos para uma alimentação saudável. Brasilia (DF); 2003.

11. Souza LJ, Gicovate Neto C, Chalita FEB, Reis AFF, Bastos DA, Souto Filho JTD, Souza, TF, Côrtes VA. Prevalência de obesidade e fatores de risco cardiovascular em Campos, Rio de Janeiro. Ara Bras Endocrinol Metab 2003 dez; 47(6).

12. Consenso Latino Americano de Obesidade. Ara Bras Endocrinol Metab 1999; 43: 21(21-67).

13. Ministério da Saúde (BR). Plano de reorganização da atenção à hipertensão arterial e no Diabetes Mellitus [CD-ROM]. Brasilia (DF); 2002.

14. Ministério da Saúde (BR). Programa Harvard-Joslin-SBD. Brasilia (DF); 1996. 